\title{
Cardiotoxicity evaluation using human embryonic stem cells and induced pluripotent stem cell-derived cardiomyocytes
}

\author{
Qi Zhao ${ }^{1 \dagger}$, Xijie Wang ${ }^{1 \dagger}$, Shuyan Wang ${ }^{1}$, Zheng Song ${ }^{1}$, Jiaxian Wang ${ }^{2}$ and Jing Ma ${ }^{1 *}$
}

\begin{abstract}
Background: Cardiotoxicity remains an important concern in drug discovery. Human pluripotent stem cell-derived cardiomyocytes (hPSC-CMs) have become an attractive platform to evaluate cardiotoxicity. However, the consistency between human embryonic stem cell-derived cardiomyocytes (hESC-CMs) and human induced pluripotent stem cell-derived cardiomyocytes (hiPSC-CMs) in prediction of cardiotoxicity has yet to be elucidated.

Methods: Here we screened the toxicities of four representative drugs (E-4031, isoprenaline, quinidine, and haloperidol) using both hESC-CMs and hiPSC-CMs, combined with an impedance-based bioanalytical method.

Results: It showed that both hESC-CMs and hiPSC-CMs can recapitulate cardiotoxicity and identify the effects of wellcharacterized compounds.

Conclusions: The combined platform of hPSC-CMs and an impedance-based bioanalytical method could improve preclinical cardiotoxicity screening, holding great potential for increasing drug development accuracy.
\end{abstract}

Keywords: Stem cells, Cardiomyocytes, Cardiotoxicity, Pharmacology

\section{Background}

Cardiac toxicity remains a major cause of restriction or withdrawal of drugs from the market [1]. Between 1990 and 2001, eight non-cardiovascular drugs were withdrawn at an estimated cost of $\$ 12$ billion due to druginduced arrhythmias [2]. Indeed, it is estimated that up to $90 \%$ of compounds that pass pre-clinical screening fail at clinical trial level, with cardiotoxicity accounting for $45 \%$ alone [3]. Current in vitro cardiotoxicity screenings rely on the artificial expression of a single hERG channel in Chinese hamster ovary or human embryonic kidney cells, based on the two guidelines ICH S7B and ICH E14 $[4,5]$. Yet, the sole reliance on simplified model cell lines, possessing interspecies differences in ion channels, biological pathways, and pharmacokinetic properties, is

\footnotetext{
* Correspondence: forapplew@163.com

${ }^{\dagger}$ Equal contributors

${ }^{1}$ China State Institutes of Pharmaceutical Industry National Shanghai Center for New Drug Safety Evaluation and Research, Shanghai, China

Full list of author information is available at the end of the article
}

putting human lives at risk. The human embryonic stem cell/human induced pluripotent stem cell (hESC/hiPSC)derived cardiomyocytes, expressing cardiac-specific factors and structural proteins, provide an alternative model for drug screening in vitro.

In combination with state-of-the-art bioanalytical methods, hESC-CMs have been reported as an alternative model for in vitro cardiotoxicity [6, 7]. Navarrete and colleagues [8] reported that hiPSC-CMs can recapitulate drug-induced arrhythmias. Although hESC-CMs and hiPSC-CMs have been reported possessing comparable ultrastructural features [9], their responses to identical compounds have not been systemically compared. The xCELLigence Real-Time Cell Analysis (RTCA) Cardio system, which utilizes impedance technology to quantify CM-beating properties, has been previously reported as an emerging method to quantify cardiac contractility [10]. In this study, we compared the capacity of hESC- and hiPSC-CMs for prediction of 
cardiotoxicity on four documented compounds, by examination with the RTCA Cardio System.

\section{Methods}

Human ESC- and iPSC-derived cardiomyocytes

Human ESC-CMs were kindly provided by the Institute of Biophysics of the Chinese Academy of Sciences, Beijing, China. Human iPSC-CMs, provided by a domestic manufacturer (Cellapy: CA2001106, Beijing, China), were also examined. Cells were incubated in maintaining medium at $37{ }^{\circ} \mathrm{C}, 5 \% \quad \mathrm{CO}_{2}$, with culture medium refreshed every 2 days. Cells were dissociated and replated into 96-well E-plates (ACEA Biosciences, Hangzhou, China), which was pre-coated with $0.1 \%$ gelatin. Cells were plated at a density of 50,000 cells per well.

\section{Real-time impedance-based bioanalyses of hPSC-derived} cardiomyocytes

Spontaneous cardiomyocytes contraction and cell health were monitored in real-time by impedance using xCELLigence real-time cellular analyzer (RTCA) Cardio system (ACEA Biosciences, Hangzhou, China). Impedance signals were monitored and recorded with $20 \mathrm{~s}$ recording per sweep.

\section{Chemicals}

Four compounds were investigated: quinidine (SigmaAldrich, St. Louis, MO, USA BCBB6721V1; 100 mmol/L stock solution in DMSO); isoproterenol (Sigma-Aldrich BCBFF4079V; $10 \mathrm{mmol} / \mathrm{L}$ stock solution in DMSO); haloperidol (Sigma-Aldrich SLBD0516; $10 \mathrm{mmol} / \mathrm{L}$ stock solution in DMSO), and E-4031 (Sigma-Aldrich 086K4616V;
$0.03 \mathrm{mmol} / \mathrm{L}$ stock solution in DMSO). Drug dilutions were equilibrated in a $37{ }^{\circ} \mathrm{C}, 5 \% \mathrm{CO}_{2}$ incubator for $30 \mathrm{~min}$ before being applied to E-plates.

\section{Data analysis and statistics}

For data analysis, cellular impedance index, beat rate, and amplitude were measured off-line using RTCA Cardio software 1.0 and normalized for each well to the baseline (pre-dose) values measured prior to the compounds treatment. Statistics were performed using SPSS 21.0 (IBM Corp., Armonk, NY, USA). Data were presented as mean \pm SEM. Statistical significance of differences was estimated by one-way ANOVA or Student's $t$ test. $p<0.05$ was considered significant.

\section{Results}

HPSC-CMs exhibit a functional cardiomyocytes phenotype in culture

Spontaneous contracting cardiomyocytes were observed using light microscopy of both hESC- and hiPSC-CMs. The cell index $(\mathrm{CI})$ value, which represents the viability of hPSC-CMs, was greatly affected by medium change (Fig. 1a-d).The spontaneous beating rate of hiPSC-CMs was stable at around $100 \mathrm{bpm}$, with rhythmic irregularity less than $10 \%$. On the other hand, hESC-CMs possessed a beating rate around $30 \mathrm{bpm}$, with rhythmic irregularity less than $30 \%$.

\section{The effects of E-4031 on hESC- and hiPSC-CMs}

E-4031 investigation was performed using various concentrations $(10,30,100$, and $300 \mathrm{nmol} / \mathrm{L})$, among which 100 and $300 \mathrm{nmol} / \mathrm{L} \mathrm{E-4031} \mathrm{substantially} \mathrm{re-}$ duced the $\mathrm{CI}$ of hiPSC-CMs (Fig. 2a) and gradually

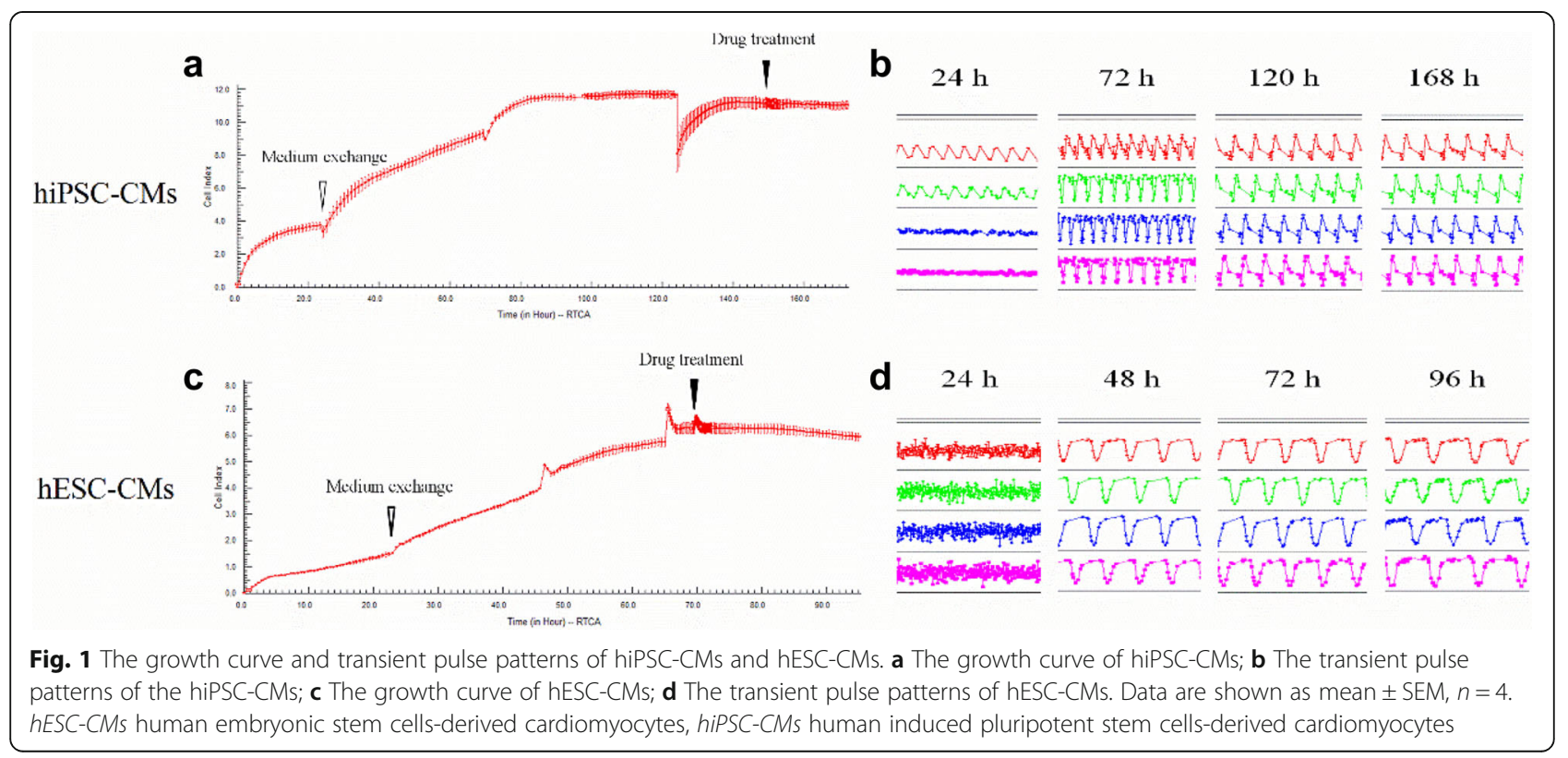




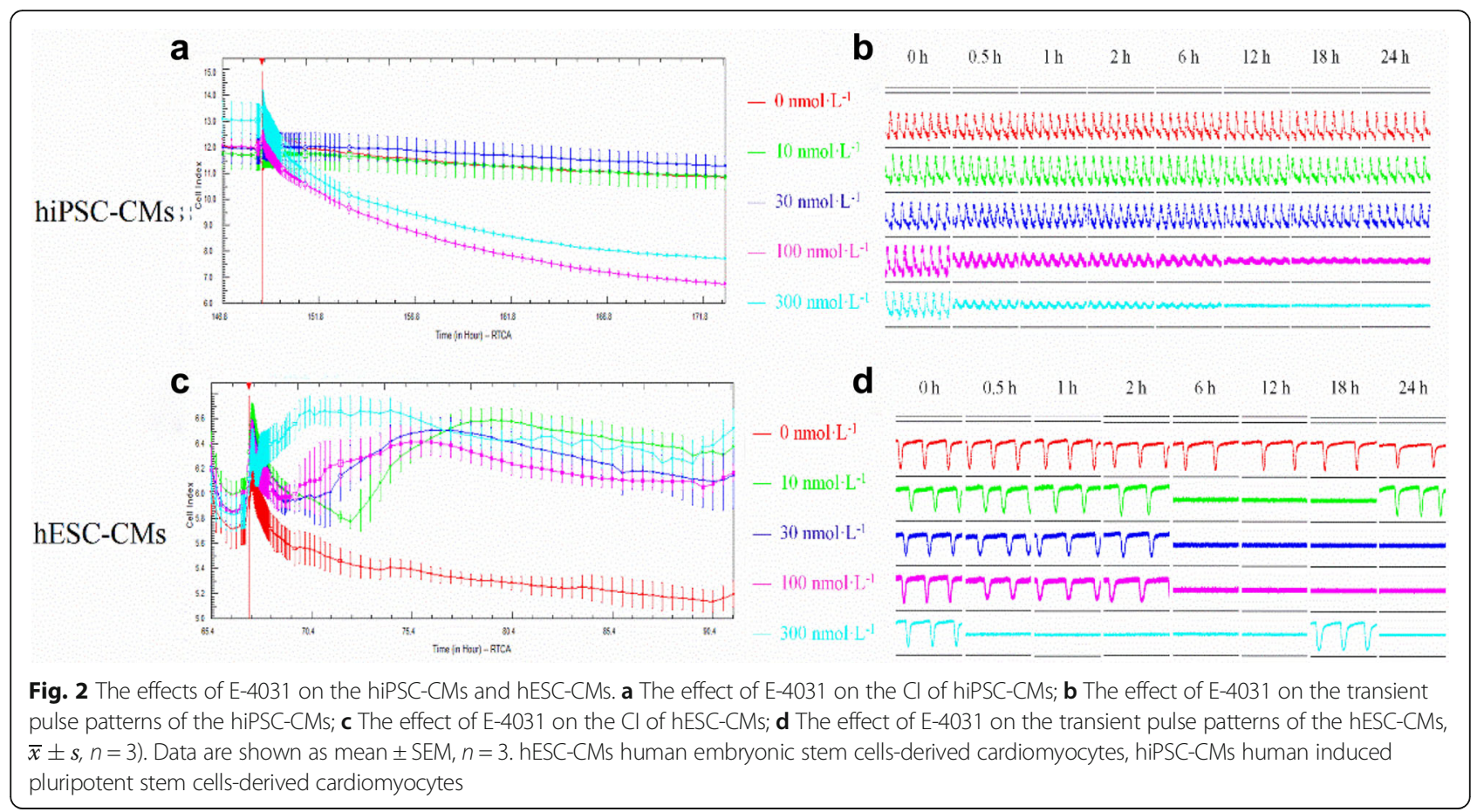

weakened the pulse signals (Fig. 2b). Spontaneous beating rates of hiPSC-CMs were markedly reduced by $100 \mathrm{nmol} / \mathrm{L} 6 \mathrm{~h}$ after administration and ceased at $12 \mathrm{~h}$. On the other hand, $300 \mathrm{nmol} / \mathrm{L}$ significantly reduced the spontaneous beating rate $1 \mathrm{~h}$ after administration and abolished spontaneous activity $2 \mathrm{~h}$ post administration. The amplitudes of $\mathrm{CI}$ were also reduced in parallel with the decrease of spontaneous rhythms. Reduction of $\mathrm{CI}$ amplitude was also observed in low concentration $(30 \mathrm{nmol} / \mathrm{L}) \mathrm{E}-4031$ with $18 \mathrm{~h}$ culture, which tended to recover at $24 \mathrm{~h}$ post administration. Calculation of the concentration-dependent effect on hiPSC-CMs revealed a $0.04 \mu \mathrm{mol} / \mathrm{L} \quad \mathrm{IC}_{50}$ of $\mathrm{E}-4031$ (Table 1). In comparison, E-4031 increased the CI of hESC-CMs (Fig. 2c). Reduction of spontaneous beating rate was observed in all concentrations of E-4031 upon immediate administration. Ten, 30, and $100 \mathrm{nmol} / \mathrm{L}$ of E4031 ceased spontaneous activity at $6 \mathrm{~h}$ post administration, while $300 \mathrm{nmol} / \mathrm{L}$ of E-4031 ceased spontaneous beating at $0.5 \mathrm{~h}$ post administration (Fig. $2 \mathrm{~d}$ ). Cells recovered automaticity $24 \mathrm{~h}$ in the $10 \mathrm{nmol} / \mathrm{L}$ group. Interestingly, temporary recovery of spontaneous beating activity was observed at $18 \mathrm{~h}$ in the $300 \mathrm{nmol} / \mathrm{L}$ group for one recording. The effects of E-4031 on amplitude were also investigated. Consistently, the amplitudes also reduced in parallel with the decrease of cellular automaticity. Calculation of the concentration-dependent effect on hESC-CMs demonstrated a $0.02 \mu \mathrm{mol} / \mathrm{L}$ of $\mathrm{IC}_{50}$ of E-4031 (Table 1).

\section{The effects of quinidine on hESC- and hiPSC-CMs}

We next assessed a class I antiarrhythmic drug, quinidine, on hPSC-CMs. Investigations demonstrated that quinidine reduced the $\mathrm{CI}$ of hiPSC-CMs in a concentration-dependent manner (Fig. 3a), and weakened the amplitudes of pulse signals at concentrations of $12.5 \mu \mathrm{mol} / \mathrm{L}$ and above (Fig. 3b). Detailed trace analysis revealed $3.13 \mu \mathrm{mol} / \mathrm{L}$ quinidine markedly reduced the beating rate $0.5 \mathrm{~h}$ after administration, which spontaneously recovered after $1 \mathrm{~h}$. However, high

Table 1 The comparison of hESC-CMs with hiPSC-CMs

\begin{tabular}{|c|c|c|c|c|}
\hline \multirow[t]{2}{*}{ Drug } & \multicolumn{2}{|l|}{ hESC-CMs } & \multicolumn{2}{|l|}{ hiPSC-CMs } \\
\hline & $\mathrm{Cl}\left(\mu \mathrm{mol} \cdot \mathrm{L}^{-1}\right)$ & $\mid \mathrm{C}_{50} / \mathrm{EC}_{50}\left(\mu \mathrm{mo} \mid \cdot \mathrm{L}^{-1}\right)$ & $\mathrm{Cl}\left(\mu \mathrm{mo} \cdot \mathrm{L}^{-1}\right)$ & $\mid C_{50} / E C_{50}\left(\mu \mathrm{mol} / \mathrm{L}^{-1}\right)$ \\
\hline$E-4031$ & $0.01 \uparrow$ & 0.02 & $0.1 \downarrow$ & 0.04 \\
\hline Isoprenaline & $0.1 \downarrow$ & 0.04 & $1 \downarrow$ & 0.03 \\
\hline Quinidine & $100 \downarrow$ & 2.18 & $100 \downarrow$ & 13.97 \\
\hline Haloperidol & NO & 0.14 & $10 \downarrow$ & 5.17 \\
\hline
\end{tabular}

Cl cell index, hESC-CMs human embryonic stem cells-derived cardiomyocytes, hiPSC-CMs human induced pluripotent stem cells-derived cardiomyocytes 


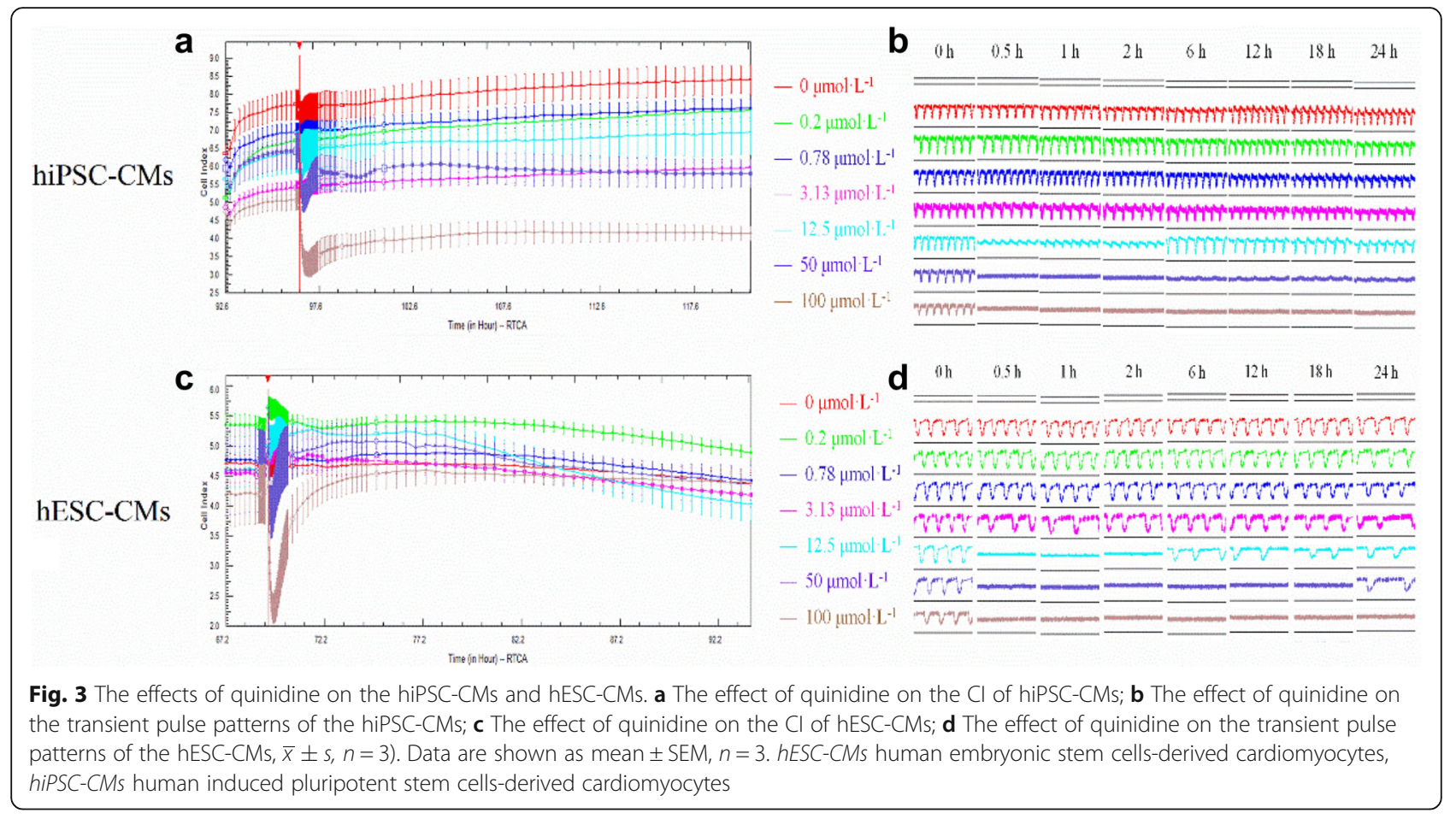

concentrations of quinidine (50 and $100 \mu \mathrm{mol} / \mathrm{L}$ ) ceased the cellular automaticity $0.5 \mathrm{~h}$ after administration without selfrecovery within the observation period. On the other hand, intermediate concentration $(12.5 \mu \mathrm{mol} / \mathrm{L})$ substantially reduced the amplitude at $0.5 \mathrm{~h}$ and $1 \mathrm{~h}$ post administration, which recovered after $2 \mathrm{~h}$. Calculation of the concentrationdependent effect on hiPSC-CMs revealed a $13.97 \mu \mathrm{mol} / \mathrm{L}$ $\mathrm{IC}_{50}$ of quinidine (Table 1). On the other hand, despite a significant temporary decrease when perfusion of $100 \mu \mathrm{mol} / \mathrm{L}$ quinidine was initiated, the $\mathrm{CI}$ of hESC-CMs was not markedly influenced by various concentrations (Fig. 3c). However, the pulse signals weakened in a concentration-dependent manner $(\geq 3.13 \mu \mathrm{mol} / \mathrm{L}$, Fig. $3 \mathrm{~d})$.The spontaneous beating rates were ceased by $12.5 \mu \mathrm{mol} / \mathrm{L}$ and $50 \mu \mathrm{mol} / \mathrm{L}$ of quinidine at $0.5 \mathrm{~h}$ post administration, which recovered at $6 \mathrm{~h}$ and $24 \mathrm{~h}$ respectively. High concentration $(100 \mu \mathrm{mol} / \mathrm{L})$ abolished the cellular automaticity without observing selfrecovery. Calculation of the concentration-dependent effect on hESC-CMs demonstrated a $2.18 \mu \mathrm{mol} / \mathrm{L}$ of $\mathrm{IC}_{50}$ of quinidine (Table 1).

\section{The effects of isoprenaline on hESC- and hiPSC-CMs}

Investigation of the positive inotropic isoprenaline demonstrated a decrease of CI of hiPSC-CMs in a concentrationdependent manner (Fig. 4a). Intermediate and high concentration of isoprenaline $(0.1,1$, and $10 \mu \mathrm{mol} / \mathrm{L})$ significantly increased the beating rate after $0.5 \mathrm{~h}$ of administration, which recovered at $24 \mathrm{~h}$ (Fig. 4b). Consistently, the amplitude of hPSC-CMs was also increased by $0.1,1$, and $10 \mu \mathrm{mol} / \mathrm{L}$ of isoprenaline. Calculation of the concentration-dependent effects on hiPSC-CMs revealed a $0.03 \mu \mathrm{mol} / \mathrm{L}$ (Table 1) $\mathrm{EC}_{50}$ of isoprenaline.

Similarly, the CI of hESC-CMs was also decreased by isoprenaline at concentrations $\geq 0.1 \mu \mathrm{mol} / \mathrm{L}$ (Fig. 4c). Spontaneous beating rate was increased $0.5 \mathrm{~h}$ post injection of isoprenaline $(0.01 \mu \mathrm{mol} / \mathrm{L})$ and recovered after $12 \mathrm{~h}$. Cellular automaticity of hESC-CMs was significantly increased by intermediate and high concentration of isoprenaline $(0.1,1$, and $10 \mu \mathrm{mol} / \mathrm{L}) 0.5 \mathrm{~h}$ post injection, and recovered at $24 \mathrm{~h}$. Calculation of the concentration-dependent effects on hESC-CMs demonstrated a $0.04 \mu \mathrm{mol} / \mathrm{L} \quad \mathrm{EC}_{50}$ of isoprenaline (Table 1).

\section{The effects of haloperidol on hESC- and hiPSC-CMs}

Haloperidol reduced the CIs and pulse signals of both hESC- and hiPSC-CMs in a concentration-dependent manner (Fig. 5a and c). Mild reduction of spontaneous beating rate was observed upon perfusing hPSC-CMs with $0.1 \mu \mathrm{mol} / \mathrm{L}$ haloperidol, which recovered at $18 \mathrm{~h}$ post administration. Haloperidol at $1 \mu \mathrm{mol} / \mathrm{L}$ significantly reduced the beating rate after $0.5 \mathrm{~h}$ upon administration, and haloperidol at $10 \mu \mathrm{mol} / \mathrm{L}$ ceased the cellular automaticity at $0.5 \mathrm{~h}$. Although self-recovery was observed in the $10 \mu \mathrm{mol} / \mathrm{L}$ group at $24 \mathrm{~h}$, the amplitude was significantly decreased compared with controls. Calculation of the concentration-dependent effects on hPSC-CMs demonstrated a $5.17 \mu \mathrm{mol} / \mathrm{L} \mathrm{IC} 50$ of haloperidol (Table 1). Consistently, mild reduction of the beating rate was observed 


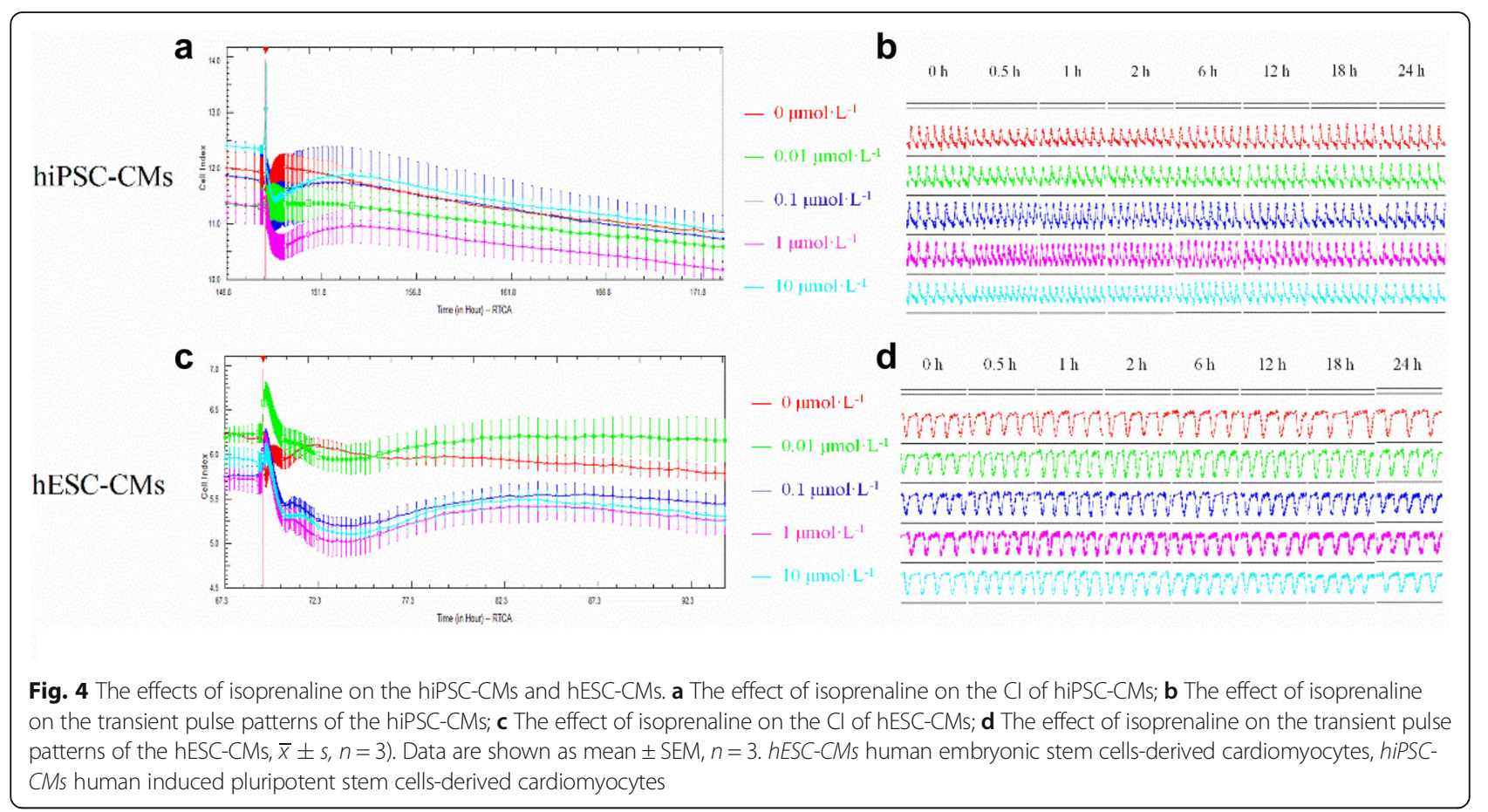

at $6 \mathrm{~h}$ in hESC-CMs post administration of low concentration of haloperidol $(0.01 \mu \mathrm{mol} / \mathrm{L})$. Haloperidol at $0.1 \mu \mathrm{mol} / \mathrm{L}$ significantly reduced the spontaneous beating rate and amplitudes of hESC-CMs. When the concentration was further increased to $1 \mu \mathrm{mol} / \mathrm{L}$, cellular automaticity was ceased at $0.5 \mathrm{~h}$, and partial recovery was observed at $24 \mathrm{~h}$ post administration. On the other hand, high concentration $(10 \mu \mathrm{mol} / \mathrm{L})$ of haloperidol abolished the spontaneous beating of hESC-CMs and no self-recovery was observed during the investigation period. Calculation of the concentration-dependent effects on hESC-CMs demonstrated a $0.14 \mu \mathrm{mol} / \mathrm{L} \mathrm{IC}_{50}$ of haloperidol (Table 1 ).

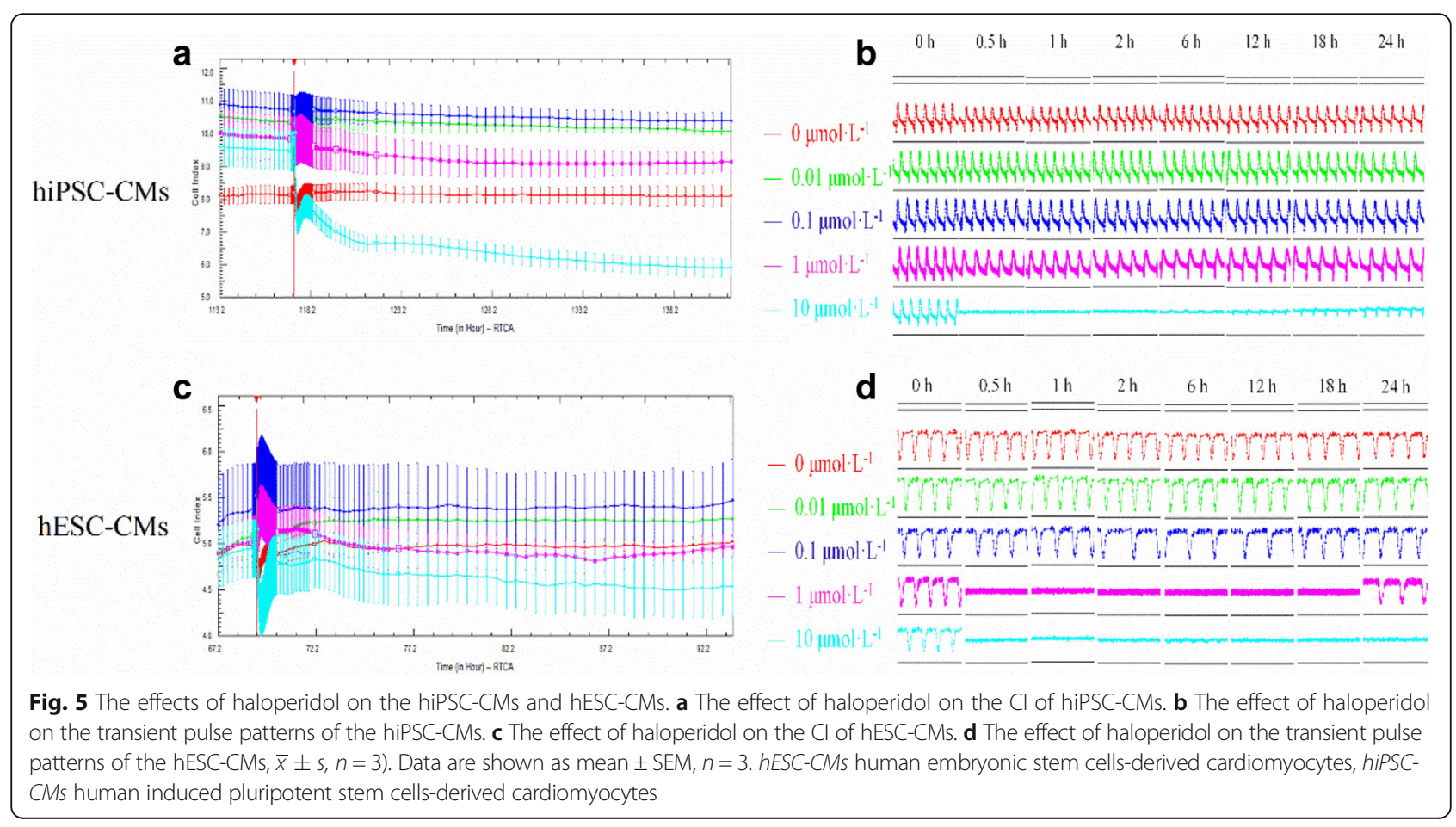




\section{Discussion}

The discovery of hPSC-CMs, including hPSC-CMs and hESC-CMs, has been an attractive in vitro model to study cardiotoxicity. Many researchers have used hiPSC$\mathrm{CMs}$ and hESC-CMs as screening models for proarrhythmic compounds during drug development to circumvent the lack of sufficient and healthy human cardiac tissue [11-15]. Here we reported the combination of an RTCA Cardio system and hPSC-CMs as a highthroughput platform, enabling unbiased real-time kinetic data acquisition for a more accurate evaluation of the cardiotoxicity of various compounds. This system can continuously monitor the contractility of cardiomyocytes by using noninvasive impedance readout and obtain quantitative data by converting impedance into cell index values [16]. In the current study, hESC-and hiPSC-CMs illustrated comparable baseline parameters before being subjected to different compounds. However, while the beating rate of the commercially available hiPSC-CMs was similar to the native human heart rate, the beating rate of the homemade hESCCMs used in this study was much lower. The spontaneous rhythm of hiPSC-CMs tended to be more consistent and less irregular along with cultivation, compared with hESC-CMs.

While conventional methods such as the manual patch clamp provide detailed information of the electrical activity of cardiomyocytes, the complexity of such technique necessitates the professional training of the electrophysiologist. In addition, the invasive nature of the patch clamp makes it difficult to perform long-term investigations on the same cells. In contrast, the current platform substantially reduced operator skill requirements compared with the patch clamp. Using the current platform, we have demonstrated recapitulation of well-characterized drug effects in both hESC- and hiPSC-CMs. We first tested E-4031, a class III antiarrhythmic drug. Our data showed good agreement with a previous study [17], which showed that the inhibition rate of $1 \mu \mathrm{mol} / \mathrm{L}$ E-4031 on hERG channel was 99.3\%. We further investigated another typical antiarrhythmic drug, quinidine, which has been reported to cause severe arrhythmia due to weak inhibition to potassium currents [11]. Using the RTCA Cardio system, we quantified the effect of quinidine on the contractility of hiPSC-CMs and hESC-CMs. Quinidine reduced the CI value of hiPSC-CMs and hESC-CMs at the same concentration. Moreover, quinidine significantly affected hiPSC-CMs and $\mathrm{hESC}$-CMs at the same concentration and the $\mathrm{IC}_{50}$ of quinidine for both cells were similar in terms of contractility and beating rhythm. We next investigated isoprenaline, a nonspecific $\beta$-agonist, which has been shown to strengthen myocardial contractility, accelerate conduction, and increase beating rate [18]. We measured the effects of isoprenaline on the contractility of hiPSC$\mathrm{CMs}$ and hESC-CMs. Isoprenaline significantly increased the contractility of hiPSC-CMs and hESC-CMs at the same concentration. Moreover, isoprenaline significantly increased both the contraction rhythm and amplitude of hiPSC-CMs and hESC-CMs at the same concentration, with similar $\mathrm{EC}_{50}$ for both cells. Beside cardiovascular drugs, we next move on to haloperidol, a dopaminergic receptor inhibitor used as an anti-schizophrenia drug. Indeed, several studies have reported that cardiotoxicity is the most serious adverse reaction of haloperidol. An overdose of haloperidol can cause QT interval prolongation, trigger TdPs, and cause sudden death in severe cases $[19,20]$. Studies have shown that haloperidol can reduce myocardial intracellular free magnesium ion levels and inhibit potassium channels, which may be the mechanism of its cardiotoxicity [21-25]. Here, we quantified the effects of haloperidol on the contractility of hiPSC-CMs and hESC-CMs. First, the CI values of hiPSC-CMs and hESC$\mathrm{CMs}$ were reduced by haloperidol at the same concentration. Furthermore, we also showed that both the beating rhythm and amplitude of hiPSC-CMs and hESC-CMs were reduced by haloperidol at the same concentration.

Overall, we have shown that the current platform enables quantitative prediction of the potential cardiotoxicity of drugs at different time points by analyzing the changes of $\mathrm{CI}$, beating rate, and amplitude of myocardial cells. We believe this method may reduce animal testing and improve both throughput and translational relevance of early cardiac safety screening by using hPSCCMs. At the same time, researchers can accurately and sensitively detect the cardiotoxicity of cardiovascular drugs and non-cardiovascular drugs with a shorter experimental period and less compounds at the earliest stages of drug development by using this method, which may result in lower attrition at the expensive late stages of the drug discovery process. However, a key limitation is that hPSC-CMs are functionally and structurally immature in multiple aspects, such as slow conduction of electrical impulses, underdeveloped sarcomeres, and poor electrophysiological properties. Therefore, future effects should be made to promote the maturation of in vitro hPSC-CMs for highly efficient and reproducible cardiotoxicity prediction.

\section{Conclusions}

In summary, we found that hESC-CMs and hiPSC-CMs of different sources present similar responses to multiple drugs. hPSC-CMs can properly predict the cardiotoxicity of different compounds. Combining with the xCELLigence Real-Time Cell Analyzer, hPSC-CMs hold the potential to increase drug development accuracy and improve upon industry standards. 


\section{Abbreviations}

Cl: Cell index; hESC-CMs: Human embryonic stem cells-derived cardiomyocytes; hiPSC-CMs: Human induced pluripotent stem cells-derived cardiomyocytes; hPSC-CMs: Human pluripotent stem cells-derived cardiomyocytes; ICH: International Conference on Harmonization; RTCA: xCELLigence ${ }^{\circledR}$ ) Real-Time Cell Analysis

\section{Acknowledgements}

The authors also would like to thank Dr. Yue Ma, Caiyun Wang and Dr. Peng Xu for their technical and critical comments on the manuscript.

\section{Funding}

This study was supported by the National Foundation of Science and Technology (2012ZX09302002)

\section{Availability of data and materials}

The data that support the findings of this study are available from the corresponding author upon reasonable request.

\section{Authors' contributions}

JM, QZ and XW designed the research. QZ, XW, SW and ZS performed the experiments. QZ and XW prepared all figures. QZ, XW and JW wrote the main manuscript text. JW revised the manuscript text, figures and provided scientific suggestions. All authors analyzed data and reviewed the manuscript. All authors read and approved the final manuscript.

\section{Competing interests}

Dr. Jiaxian Wang is one of the co-founders of Help Stem Cell Innovations (www.helpsci.com.cn).

\section{Consent for publication}

Not applicable.

\section{Ethics approval and consent to participate}

hiPSC-CMs were obtained from Beijing Cellapy Biotechnology Co., Ltd. hESCCMs were obtained from the Institute of Biophysics of the Chinese Academy of Sciences. In both cases, informed consent was obtained from donors and in compliance with all national regulations

\section{Author details}

${ }^{1}$ China State Institutes of Pharmaceutical Industry National Shanghai Center for New Drug Safety Evaluation and Research, Shanghai, China. ${ }^{2}$ Department of Cardiology, the First Affiliated Hospital of Nanjing Medical University, Nanjing Medical University, Nanjing, China.

Received: 20 November 2016 Revised: 12 December 2016 Accepted: 27 December 2016 Published online: 09 March 2017

\section{References}

1. Kannankeril PJ, Roden DM. Drug-induced long QT and torsade de pointes: recent advances. Curr Opin Cardiol. 2007;22(1):39-43.

2. Fermini B, Fossa AA. The impact of drug-induced $\mathrm{QT}$ interval prolongation on drug discovery and development. Nat Rev Drug. 2003;2(6):439-47.

3. Kola I, Landis J. Can the pharmaceutical industry reduce attrition rates? Nat Rev Drug Discov. 2004;3(8):711-5.

4. ICH Harmonised Tripartite Guideline. The non-clinical evaluation of the potential for delayed ventricular repolarization (QT interval prolongation) by human pharmaceuticals. S7B.2005. Available from: www.ich.org/products/ guidelines/safety/article/safety-guidelines.html. Accessed Oct 2014.

5. ICH Harmonised Tripartite Guideline. The clinical evaluation of QT/QTc interval prolongation and proarrhythmic potential for non-antiarrhythmic drugs. E14. 2005. Available from: www.ich.org/fileadmin/Public_Web_Site/ICH_Products/ Guidelines/Efficacy/E14/E14_Guideline.pdf. Accessed Oct 2014.

6. Mandenius CF, Steel D, Noor F, Meyer T, Heinzle E, Asp J, et al. Cardiotoxicity testing using pluripotent stem cell-derived human cardiomyocytes and state-of-the-art bioanalytics: a review. J Appl Toxicol. 2011;31(3):191-205.

7. Clements M. High-throughput multi-parameter profiling of electrophysiological drug effects in human embryonic stem cell derived cardiomyocytes using multi-electrode arrays. Toxicol Sci. 2014;140(2):445-61.

8. Navarrete EG, Liang P, Lan F, Sanchez-Freire V, Simmons C, Gong T, et al, Screening drug-induced arrhythmia using human induced pluripotent stem cell-derived cardiomyocytes and low-impedance microelectrode arrays. Circulation. 2013;128(11 Suppl 1):S3-13.

9. Gherghiceanu M, Barad L, Novak A, Reiter I, Itskovitz-Eldor J, Binah O, et al. Cardiomyocytes derived from human embryonic and induced pluripotent stem cells: Comparative ultrastructure. J Cell Mol Med. 2011;15(11):2539-51.

10. Scott CW, Zhang X, Abi-Gerges N, Lamore SD, Abassi YA, Peters MF. An impedance-based cellular assay using human iPSC-derived cardiomyocytes to quantify modulators of cardiac contractility. Toxicol Sci. 2014;142(2):331-8.

11. Braam SR, Tertoolen L, Stolpe AVD, Meyer T, Passier R, Mummery CL. Prediction of drug-induced cardiotoxicity using human embryonic stem cell-derived cardiomyocytes. Stem Cell Res. 2010;4(2):107-16.

12. Caspi O, Itzhaki I, Kehat I, Gepstein A, Arbel G, Huber I, et al. In vitro electrophysiological drug testing using human embryonic stem cell derived cardiomyocytes. Stem Cells Dev. 2009;18(1):161-72.

13. Itzhaki I, Maizels L, Huber I, Zwi-Dantsis L, Caspi O, Winterstern A, et al. Modelling the long QT syndrome with induced pluripotent stem cells. Nature. 2011;471(471):225-9.

14. Jonsson MK, Duker G, Tropp C, Andersson B, Sartipy P, Vos MA, et al. Quantified proarrhythmic potential of selected human embryonic stem cell-derived cardiomyocytes. Stem Cell Res. 2010;4(3):189-200.

15. Moretti A, Bellin M, Welling A, Jung CB, Lam JT, Bott-Flügel $L$, et al. Patient-specific induced pluripotent stem-cell models for long-QT syndrome. N Engl J Med. 2010;363(15):1397-409.

16. Xi B, Wang T, Li N, Ouyang W, Zhang W, Wu J, et al. Functional cardiotoxicity profiling and screening using the xCELLigence RTCA cardio system. J Lab Autom. 2011;16(6):415-21.

17. Cardiac Channel Panel. Assays and Drug Mechanisms of Action [JR/OL] (2011-02-09). http://www.chantest.com/screening-services/safety/cardiac-apa/ drug-mechanism-of-action. Accessed 9 Feb 2011.

18. Nguemo F, Šarić T, Pfannkuche K, Watzele M, Reppel M, Hescheler J. In vitro model for assessing arrhythmogenic properties of drugs based on highresolution impedance measurements. Cell Physiol Biochem. 2012;29(5-6):819-32.

19. Fayer SA. Torsades de pointes ventricular tachyarrhythmia associated with haloperidol. J Clin Psychopharmacol. 1986;6(6):375-6.

20. Hunt N, Stern TA. The association between intravenous haloperidol and Torsades de Pointes: three cases and a literature review. Psychosomatics. 1995;36(6):541-9.

21. Jabotinsky-Rubin K, Durst R, Levitin LA, Moscovich DG, Silver H, Lerner J, et al. Effects of haloperidol on human plasma magnesium. J Psychiatr Res. 1993;27(2):155-9.

22. Satoh Y, Sugiyama A, Tamura K, Hashimoto K. Effect of magnesium sulfate on the haloperidol-induced QT prolongation assessed in the canine in vivo model under the monitoring of monophasic action potential. Jpn Circ J. 2000;64(6):445-51.

23. Yang SB, Proks P, Ashcroft FM, Rupnik M. Inhibition of ATP-sensitive potassium channels by haloperidol. Br J Pharmacol. 2004;143(8):960-7.

24. Bebarova M, Matejovic PM, Novakova M. Effect of haloperidol on transient outward potassium current in rat ventricular myocytes. Eur J Pharmacol. 2006;550(1-3):15-23.

25. Takahashi K, Tanabe K, Ohnuki M, Narita M, Ichisaka T, Tomoda K, et al. Induction of pluripotent stem cells from adult human fibroblasts by defined factors. Cell. 2007;131(3):861-72.

\section{Submit your next manuscript to BioMed Central and we will help you at every step:}

- We accept pre-submission inquiries

- Our selector tool helps you to find the most relevant journal

- We provide round the clock customer support

- Convenient online submission

- Thorough peer review

- Inclusion in PubMed and all major indexing services

- Maximum visibility for your research

Submit your manuscript at www.biomedcentral.com/submit
) Biomed Central 